\title{
Analysis of 2D GISAXS patterns obtained on semiconductor nanocrystals
}

\author{
M. Buljan ${ }^{\mathrm{a}, *}$, K. Salamon ${ }^{\mathrm{b}}$, P. Dubcek ${ }^{\mathrm{a}}$, S. Bernstorff ${ }^{\mathrm{c}}$, I.D. Desnica-Frankovic ${ }^{\mathrm{a}}$, \\ O. Milat ${ }^{\mathrm{b}}$, U.V. Desnica ${ }^{\mathrm{a}}$ \\ ${ }^{a}$ R. Boskovic Institute, P.O. Box 180, 10001 Zagreb, Croatia \\ ${ }^{\mathrm{b}}$ Institute of Physics, Bijenicka c. 52, 10000 Zagreb, Croatia \\ ${ }^{\mathrm{c}}$ Sincrotrone Trieste, SS 14 km163,5, 34012 Basovizza, Italy
}

Received 16 June 2002; received in revised form 20 August 2002; accepted 30 September 2002

\begin{abstract}
Grazing incidence small-angle X-ray scattering (GISAXS) was applied in the study of semiconductor nanocrystals embedded in a light matrix. The appropriate mathematical apparatus was developed, so that a full characterization of 3D ensemble of nanoparticles, formed in the implanted layer, can be obtained from GISAXS spectra recorded on twodimensional (2D) detector.

The investigated $\mathrm{CdS}$ nanocrystals in $\mathrm{SiO}_{2}$ substrate were formed by ion beam synthesis and subsequent annealing at $1273 \mathrm{~K}$. From the fits to the theoretical expressions, the average particle diameter, the shape, as well as the size distribution were determined. The obtained results are in good agreement with TEM results performed on the analogous samples.
\end{abstract}

(C) 2003 Elsevier Science Ltd. All rights reserved.

Keywords: Nanocrystals; Quantum dots; X-ray scattering; SAXS; GISAXS; Implantation; CdS

\section{Introduction}

Significant differences in the opto-electronic properties of nanoparticles as compared to their bulk analogues have put nanocrystalline materials in the midst of intense worldwide investigations. Nanocrystals show size-dependent optical and electronic properties, opting them for a variety of potential applications, which include optical filters, quantum dot lasers and high-speed nonlinear optical switches [1-4].

\footnotetext{
*Corresponding author. Tel.: 385-1-4561-072; fax: +385-14680-114.

E-mail address: mbuljan@rudjer.irb.hr (M. Buljan).
}

Grazing incidence small angle X-ray scattering (GISAXS) is a powerful technique for structural characterization of nanocrystals in the matrix. From the GISAXS pattern, which represents the statistical average over a large number of nanoparticles (about $10^{12}$ ), it is possible to determine the size, shape, volume concentration, interparticle distance as well as size distribution of nanocrystals $[5,6]$. However, to get a reliable insight into the structural properties of the investigated material, it is very important that a suitable analysis/fitting procedure be applied, as well as the proper model chosen, that will be most appropriate for a given situation. In that respect, we have developed a methodology for systematic 
analysis of GISAXS spectra obtained from a thin sub-surface layer of semiconductor quantum dots (QDs) embedded in a light matrix. In this work, the method is applied on 2D GISAXS patterns obtained from CdS nanoparticles formed in $\mathrm{SiO}_{2}$ matrix by ion beam synthesis.

\section{Experimental}

Samples were produced by implanting equal doses of $\mathrm{Cd}$ and $\mathrm{S}$ atoms in amorphous $\mathrm{SiO}_{2}$ substrates. Multiple energy implantations were chosen so as to obtain uniform concentration profiles of $\mathrm{Cd}$ and $\mathrm{S}$ atoms up to the depth of $150 \mathrm{~nm}$ for each dose, as determined by Rutherford backscattering [7]. Three different doses were implanted, giving rise to concentrations: $C 1=5.3 \times 10^{21} \mathrm{~cm}^{-3}, C 2=2.0 \times 10^{21} \mathrm{~cm}^{-3}$ and $C 3=0.8 \times 10^{21} \mathrm{~cm}^{-3}$. The subsequent annealing at $T_{\mathrm{a}}=1273 \mathrm{~K}$ for $1 \mathrm{~h}$ caused diffusion of $\mathrm{Cd}$ and $\mathrm{S}$ atoms and their fusion into CdS nanocrystals.

GISAXS measurements were performed at the ELETTRA Synchrotron radiation source in Trieste, using X-ray photon energy of $8 \mathrm{keV}$ $(\lambda=0.154 \mathrm{~nm})$. The sample was mounted on a stepper-motor-controlled tilting stage with a step resolution of $0.001^{\circ}$. For each sample, the GISAXS pattern was first recorded at the angle of incidence, $\alpha_{i}$, equal to the critical angle for total external reflection, $\alpha_{c}$. The incidence angle was then systematically increased to probe deeper parts of the layer and thus perform the depth distribution profiling.

$\mathrm{X}$-ray scattering intensity spectra were recorded with a position-sensitive two-dimensional CCD detector containing $1024 \times 1024$ pixels, positioned perpendicular to the incident beam. A thin Alstripe was placed in front of the $2 \mathrm{D}$ detector in order to attenuate the very intense specular reflected beam and thus avoid overflow of the detector. Spectra were corrected for background intensity and detector response, and then for absorption and refraction effects.

The GISAXS analysis was based on the local monodisperse approximation (LMA), in which the system is approximated as the sum of many monodisperse subsystems, and the total scattering is calculated as the sum of the scattering from the subsystems, weighted by the size distribution. In the analysis, surface contributions were modelled using distorted wave Born approximation (DWBA) [8,9].

\section{Results and discussion}

Fig. 1 depicts a characteristic 2D GISAXS spectrum acquired from a sample characterized with concentration $C 1=5.3 \times 10^{21} \mathrm{~cm}^{-3}$ of $\mathrm{Cd}$ and $\mathrm{S}$ atoms. The most pronounced feature is an interference maximum represented by an almost spherical half-ring. Quite generally, 2D-GISAXS spectra result from combined effects of X-ray scattering on the particles within the substrate and scattering from the real surface (Fig. 2a). Particle scattering is here of prime interest, since from it, structural information about particles (shape, size, interparticle distance, etc.) could be resolved. Surface roughness-induced scattering is superimposed on the signal from the particles and has to be taken into account. Only diffuse surface scattering is considered, since the coherent contribution is blocked by the beam stop [10]. The geometry of the incident, refracted and scattered beam, as well as the corrections that were taken into account, are presented in Fig. 2b. The incident beam reaches the surface at an angle $a$, and exits at the angle $\sigma .2 \theta$ is the scattering angle, related to the wave vector as

$\mathbf{q}=\mathbf{k}_{\mathbf{f}}-\mathbf{k}_{\mathbf{i}}, \quad|q|=\frac{4 \pi}{\lambda} \sin (\theta)$,

where $\mathbf{k}_{\mathbf{i}}$ and $\mathbf{k}_{\mathbf{f}}$ stand for the wave vectors of incident and exiting beam, respectively. The detector measures component of the wave vector $\mathbf{q}$ that is vertical to the surface of the sample: $\mathbf{q}_{\perp}=$ $\mathbf{q}_{\mathbf{y}}+\mathbf{q}_{\mathbf{z}}$, whereas the parallel one $\left(q_{x}\right)$ is much smaller and can be determined from the relation: $q^{2}=q_{x}^{2}+q_{y}^{2}+q_{z}^{2}$ and the scattering geometry. GISAXS spectra were first corrected for the refraction, transmission and absorption effects. For X-rays, the index of refraction of solids has a complex form and is given as $n=1-\delta-\mathrm{i} \beta . \delta$ is a parameter responsible for beam refraction, whereas $\beta$ describes absorption [10]. Since the 


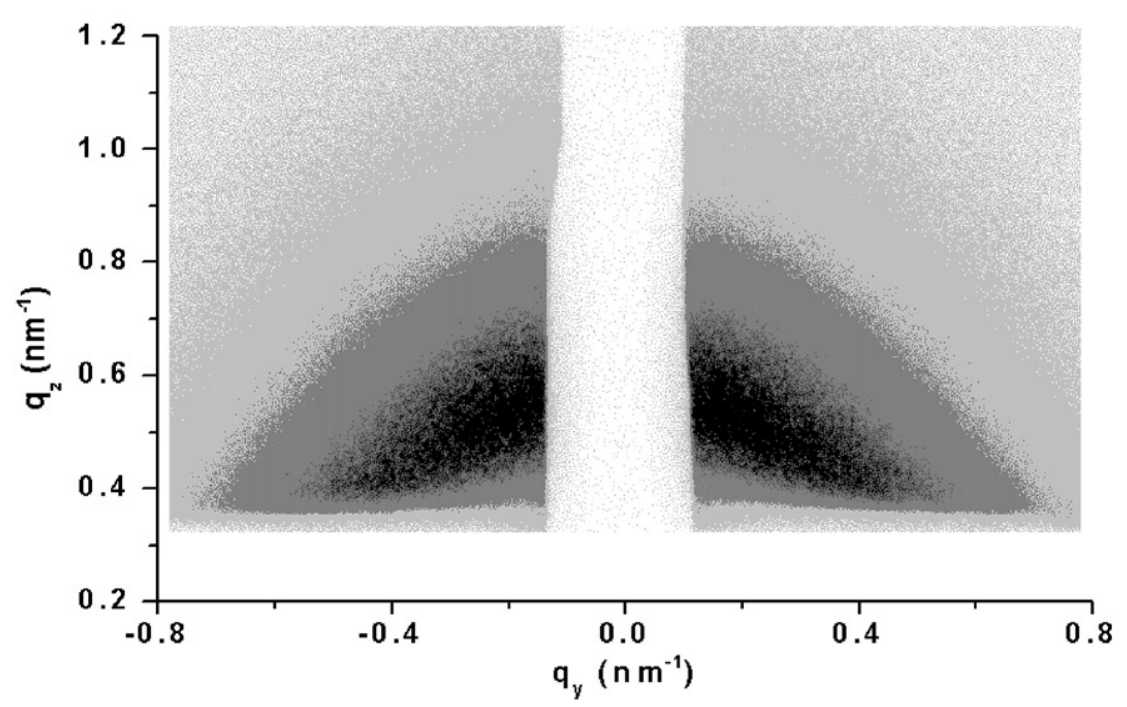

Fig. 1. 2D GISAXS spectra of the CdS nanocrystals in $\mathrm{SiO}_{2}$ substrates in concentration $C 1=5.3 \times 1021 \mathrm{~cm}^{-3}$, obtained at angle of incidence $\alpha_{\mathrm{i}}=\alpha_{\text {crit }}+0.05^{\circ}$.

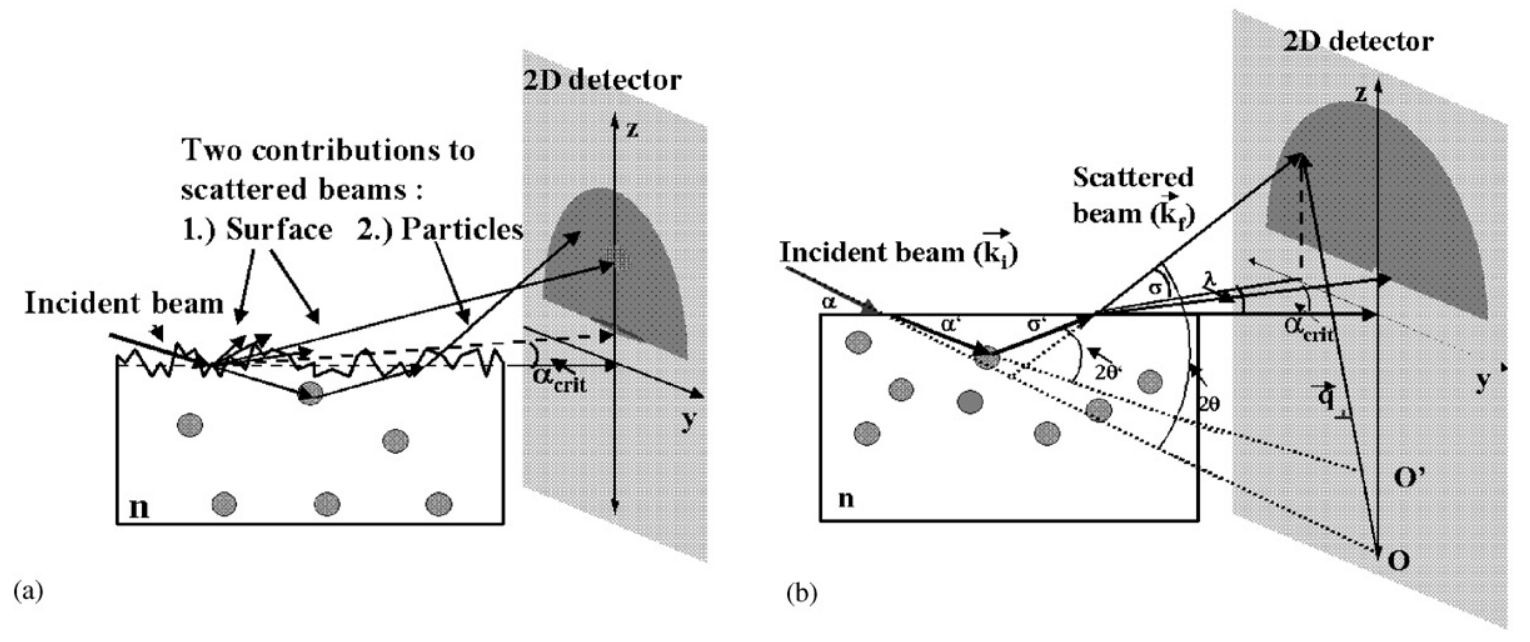

Fig. 2. (a) Two contributions to scattered beams: diffuse surface scattering and particle scattering. (b) Geometry of GISAXS, scheme of relevant angles for refraction corrections.

incident X-ray is refracted at the boundary of two materials with different refraction indices, the actual scattering angle, 20, which is being measured at the detector, differs from the real scattering angle within the sample, $2 \theta^{\prime}$. In addition, the direct beam position moves as well $\left(O \rightarrow O^{\prime}\right.$, Fig. 2b). The $2 \theta^{\prime}$ angle is determined from the scattering geometry and refraction indexes $\left(n_{\mathrm{i}}\right)$ for a given material [11]. The second effect, which also arises due to different refraction indexes, is the transmission coefficient for exiting beam, which varies depending on the incidence angle. The intensity of the transmitted beam is determined by the Fresnel transmission coefficient [12].

Fig. 3 depicts a 1D linear profile of 2D GISAXS spectrum taken along polar angle $\phi=60^{\circ}$, together with corrections applied to account for the 
effects of refraction, transmission and absorption. Each successive correction takes into account the previous one, the spectrum denoted with the full line, is being corrected for all effects.

Fig. 4 shows 1D scans, taken along different polar angles $\left(55^{\circ}, 65^{\circ}\right.$, and $\left.75^{\circ}\right)$, before (a) and after (b) the corrections for refraction, absorption and transmission effects were applied. All scans are nearly equal, indicating that the embedded nanoparticles are spherical in shape and isotropically distributed within the matrix.

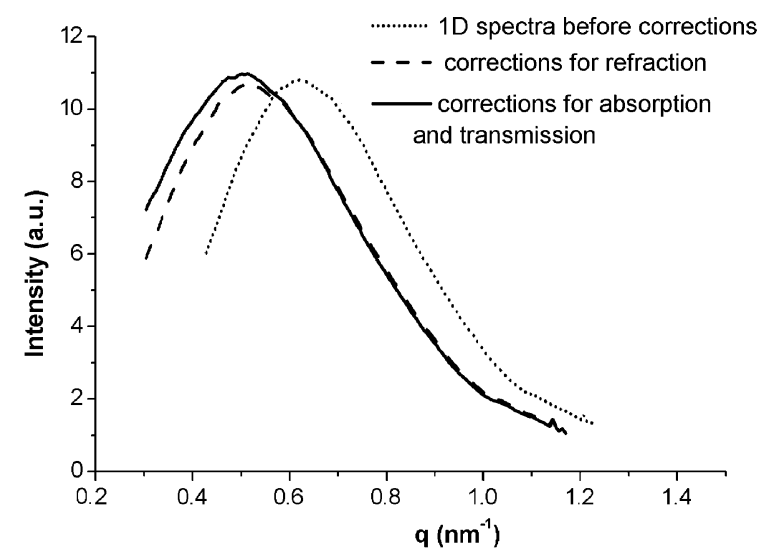

Fig. 3. Corrections for the refraction, transmission and absorption effects. Dotted line denotes spectrum prior to the correction was applied, dashed line is the spectrum corrected for the scattering angle $2 \theta$, while full line is spectrum corrected for all effects - refraction, transmission and absorption.

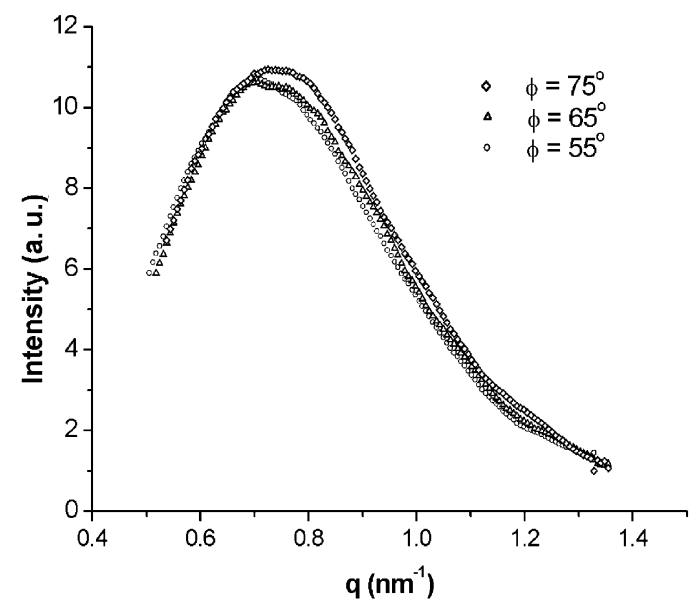

To get quantitative information, local monodisperse approximation was used. It assumes that the positions and size of particles are completely correlated, and that particles in the substrate are locally monodisperse. The scattering intensity has the form:

$I(q) \propto \int_{0}^{\infty} f(q, R) S\left(q, R_{\mathrm{HS}}, \eta_{\mathrm{HS}}\right) G(R, w)$,

where $F(q, R)$ is the form factor of a homogeneous sphere of radius $R, S\left(q, R_{\mathrm{HS}}, \eta_{\mathrm{HS}}\right)$ is the structure factor of the assembly expressed within PercusYevick approximation [10], $\eta_{\mathrm{HS}}$ is the volume fraction of the hard spheres, $G(R, w)$ is the Gaussian size distribution function, specified by its width parameter $w, G(R, w)=(1 / \sqrt{2 \pi w})$ $\exp \left(-\left(R-R_{0}\right)^{2} / 2 w^{2}\right)$.

Surface contribution was expressed within DWBA approximation. It depends on scattering geometry and parameters that characterize surface roughness [8].

Fig. 5 shows characteristic 1D scans of GISAXS patterns for polar scans at $\phi=70^{\circ}$, as well as the best LMA + DWBA fits to the experimental points for three doses of $\mathrm{Cd}$ and $\mathrm{S}$ atoms. The first maximum on graphs corresponds to the surface, and second one to the nanoparticle contributions. A different shape of 1D scans for different concentrations indicates changes in the particle properties. It could be seen that the surface

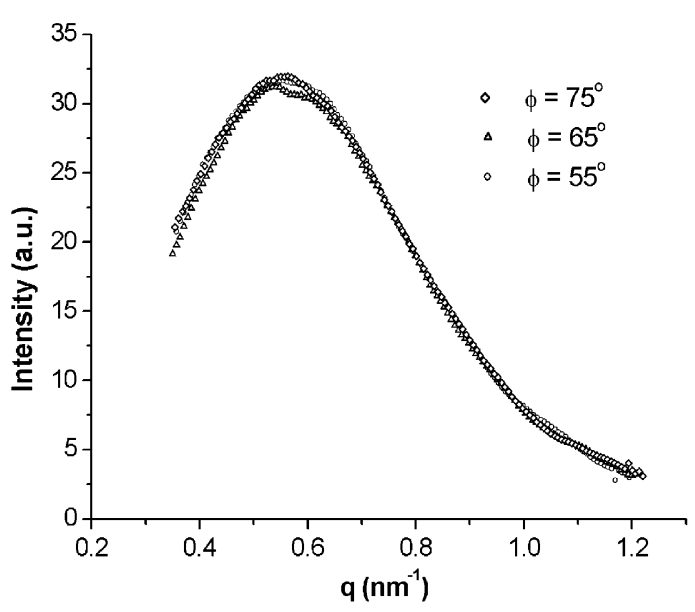

Fig. 4. (a) Line profile taken along different polar angles before corrections, (b) same profiles after corrections. 


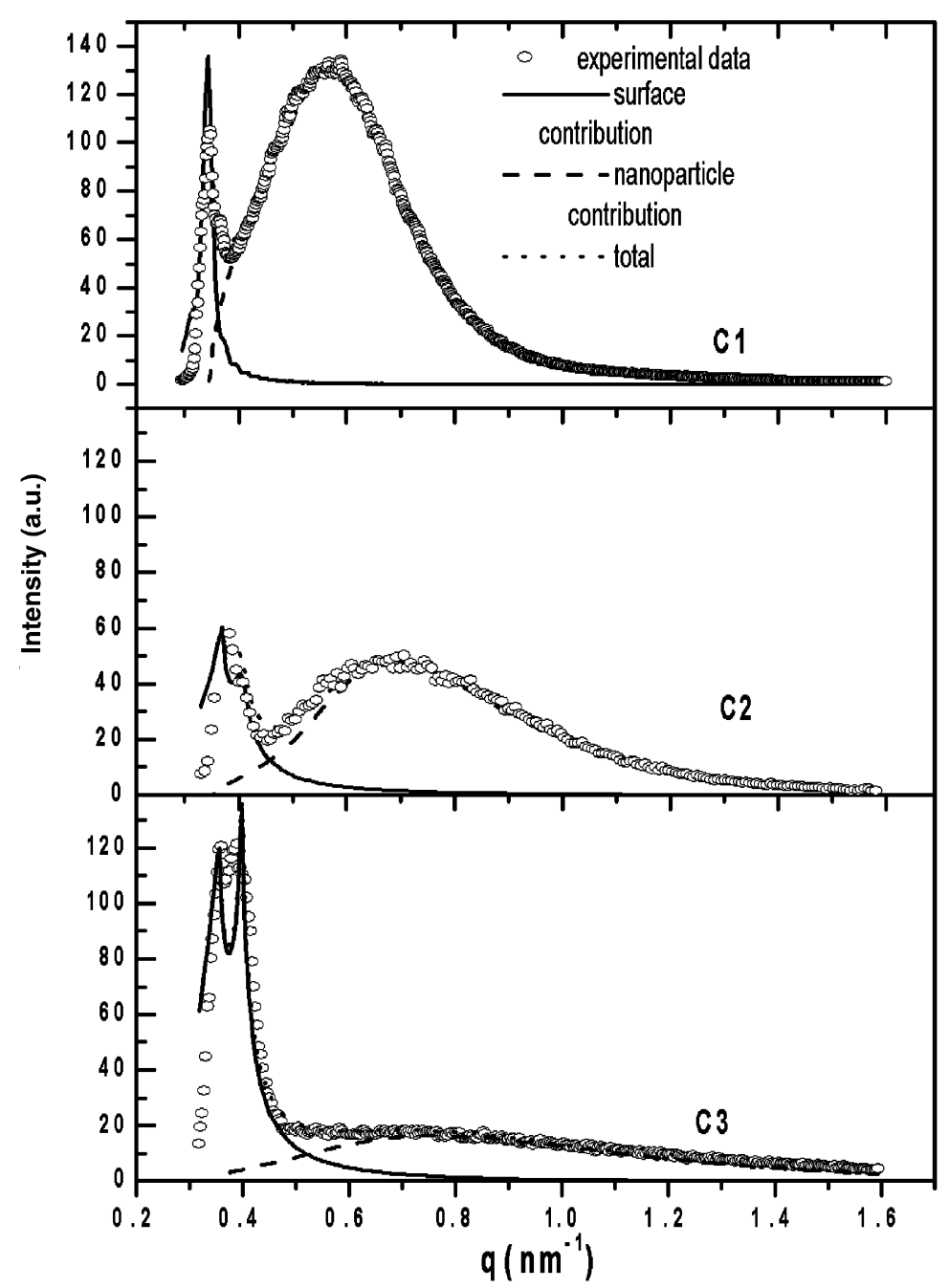

Fig. 5. Scattering curves for selected concentrations of CdS, obtained at angles of incidence $\alpha_{i}=\alpha_{\text {crit }}+0.05^{\circ}$, together with the associated fits. Full line denotes fit for the surface diffuse scattering and dashed line scattering from the particles in the substrate. Dots are experimental values.

contribution is important for smaller values of $\mathbf{q}$ and for lower concentrations (C3), where the particle-induced signal is much weaker.

The obtained average diameter $D$ of nanocrystals was determined to be $9.9 \mathrm{~nm}$, with the width of size distribution $w=2.8 \mathrm{~nm}$ for the highest dose. For the two lower doses, diameters were 4.8 and $3.2 \mathrm{~nm}$, with $w=1.9$ and $1.0 \mathrm{~nm}$, respectively, in good agreement with TEM results [3]. The average distance between nanocrystals was found to be 13.5, 7.0 and $6.4 \mathrm{~nm}$. The volume fraction $\eta$ of nanocrystals was established as $21 \%, 12 \%$, and $5 \%$, which agrees reasonably well with the expected volume fraction of CdS material in the substrate, assuming that practically all $\mathrm{Cd}$ and $\mathrm{S}$ atoms were synthesized into $\mathrm{CdS}$.

\section{Conclusion}

A methodology for the analysis of 2D-GISAXS patterns of ion-beam synthesized semiconductor 
nanocrystals in light matrix was developed and applied to $\mathrm{CdS}$ nanoparticles in $\mathrm{SiO}_{2}$ substrate. Structural information about embedded nanocrystals were obtained by fitting experimental data to the theoretical curves for particle scattering (based on LMA) and diffuse surface scattering (based on DWBA). Corrections for refraction, transmission and absorption effects were included in the LMA fit. From such analysis, we have obtained statistical information on nanoparticle shape, interparticle distance and size distribution in the film. For all three doses, the results have shown that the synthesized CdS QDs are spherical in shape, isotropically distributed in the layer, with average diameter $3.2,4.8$ and $9.9 \mathrm{~nm}$, as well as interparticle distances of $6.4,7.0$, and $13.5 \mathrm{~nm}$, respectively, both increasing with concentration of implanted ions. These results agree with TEM results obtained on analogous samples.

\section{Acknowledgements}

This research was partly supported by the Ministry of Science and Technology of Croatia.
C.W. White from Oak Ridge National Laboratory has kindly provided the samples.

\section{References}

[1] Meldrum A, Boatner LA, White CW. Nucl Instrum Methods B 2001;178:7-16.

[2] Budai JD, White CW, Withrow SP, Chisholm MF, Zhu J, Zuhr RA. Nature 1997;390:384-6.

[3] White CW, Meldrum A, Budai JD, Withrow SP, Sonder E, Zuhr RA, Hembree Jr DM, Wu M, Henderson DO. Nucl Instrum Methods B 1999;148:991-6.

[4] Karl H, Grosshans I, Attenberger W, Schmid M, Stritzker B. Nucl Instrum Methods B 2001;178:126-30.

[5] Naudon A, Thiaudiere D. J Appl Cryst 1997;30:822-7.

[6] Babonneau D, Videnović IR, Garnier MG, Oelhafen P. Phys Rev B 2001;63:195401.

[7] Meldrum A, White CW, Boatner LA, Andersen IM, Zuhr RA, Sonder E, Budai JD, Henderson DO. Nucl Instrum Methods B 1999;148:957-63.

[8] Sinha SK, Sirota EB, Garoff S, Stanley HB. Phys Rev B 1998;38:2297-311.

[9] Levine JR, Cohen JB, Chung YW, Georgopoulos P. J Appl Crystallogr 1989;22:528-32.

[10] Pedersen JS. J Appl Crystallogr 1994;27:595-608.

[11] Martorana A, Longo A, d'Acapito F, Maurizio C, Cataruzza E, Gonella F. J Appl Crystallogr 2001;34:152-6.

[12] Ping Lee. Am J Phys 1995;53:9. 